\title{
DGI-Praxistage 2015
}

\section{Alexander Klaus* \\ Informationsvermittlung: Ein Fall für visuelle Datenanalyse}

DOI 10.1515/iwp-2016-0007

Zeit ist kostbar. Das bekommt vor allem das Management in Unternehmen zu spüren. Entscheidungen müssen oft zwischen zwei Terminen getroffen werden und das setzt unter Druck. Es bedarf deshalb neuer Methoden, um komplexe Informationen so zu präsentieren, dass sie auch in kurzer Zeit leicht verständlich und nachvollziehbar sind und damit zur Grundlage der Entscheidungsfindung werden. Plattformen für Business Intelligence, die sich auch auf die Visualisierung von Daten spezialisiert haben, wurden genau für diese hohen Anforderungen konzipiert.

Es gibt Datenvisualisierungstools, die mit ihrer intuitiven Handhabung werben. Und es gibt klassische Reportingtools, die auf saubere Datenaufbereitung bauen. Unternehmen brauchen jedoch mehr als ein Tool, das Teildisziplinen - wie sorgfältiges Datenbankmanagement, Datenvisualisierung, Governed Analytics oder Self-Service abdeckt: Damit auch komplexe Informationen umfassend aber dennoch leicht verständlich vermittelt werden können, ist ein Plattformansatz erforderlich. Vom Vereinen unterschiedlicher Datenquellen und -formaten aus einem Data Lake, über das Einhalten von Governance-Richtlinien bis zur Vermittlung neuer Erkenntnissen mittels Visualisierungen - eine Visual Analytics-Plattform muss eine Bandbreite an Kriterien erfüllen:

\section{Datenvisualisierung für alle Bedürfnisse}

Das Visualisieren von Daten ist nur dann sinnvoll, wenn die Grafiken schnell und einfach verständlich sind. Das können klassische Diagramme und Graphen sein, Geovisualisierungen oder Scatterplots. Wichtig für Datenvisualisierungs-Lösungen ist der Self-Service-Gedanke: Via Drag-and-Drop müssen Visualisierungen erweitert werden

*Kontaktperson: Alexander Klaus, QlikTech GmbH, Tersteegenstraße 25, 40474 Düsseldorf,

E-Mail: alexander.klaus@qlik.com,www.qlik.com/de können und durch einen Klick muss eine Datenquelle abrufbar sein. Auch ungeübte Nutzer der Software sollen anhand von Visualisierungen schnell zu den gewünschten Informationen und neuen Erkenntnissen kommen. Außerdem müssen Visualisierungen problemlos in Websites integriert werden können und in Echtzeit teilbar sein - auch mit Kollegen oder Geschäftspartnern. Denn Mitarbeiter arbeiten niemals isoliert voneinander, Informationen werden unentwegt ausgetauscht. Datenvisualisierung muss deshalb von Anfang an auf Kollaboration ausgelegt sein.

\section{Mobiler Zugriff auf Daten}

In unserer heutigen mobilen Welt wäre jede Information nutzlos, wenn sie nicht von unterwegs abgerufen werden kann. Alle Bestandteile einer Visual Analytics Plattform egal ob einzelne Daten oder fertige Visualisierungen - müssen zu jeder Zeit und an jedem Ort verfügbar sein. Mobile Nutzung darf nicht nur möglich sein, die Lösung muss mit responsivem Design und entsprechender Menüführung speziell auf sämtliche Endgeräte ausgerichtet sein. Und dies aus mehreren Gründen: Zum einen nutzt fast jeder Mitarbeiter im Unternehmen im Laufe eines Arbeitstages mehrere unterschiedliche Geräte. Während der stationäre PC im Büro weiterhin bevorzugtes Endgerät bleibt, ist das Smartphone bei Geschäftsterminen beliebt. Zum anderen spielt der Zeitfaktor eine wichtige Rolle: Die meisten Entscheidungsträger müssen innerhalb eines Tages Entscheidungen treffen, immer unter der Voraussetzung, dass die erforderlichen Daten und Informationen schnell zur Verfügung stehen.

\section{Governance und Data Discovery}

Damit Information nicht zu einem raren Gut wird, das nur ausgewiesenen IT-Experten vorbehalten ist, müssen Visual Analytics-Lösungen intuitiv bedienbar sein. Data Discovery hat nicht nur Auswirkungen auf die Benutzeroberfläche, sondern bedeutet auch, dass Daten vom Anwender nach eigenen Bedürfnissen aus unterschiedlichen Per- 


\section{Qlik Visual Analytics Platform}

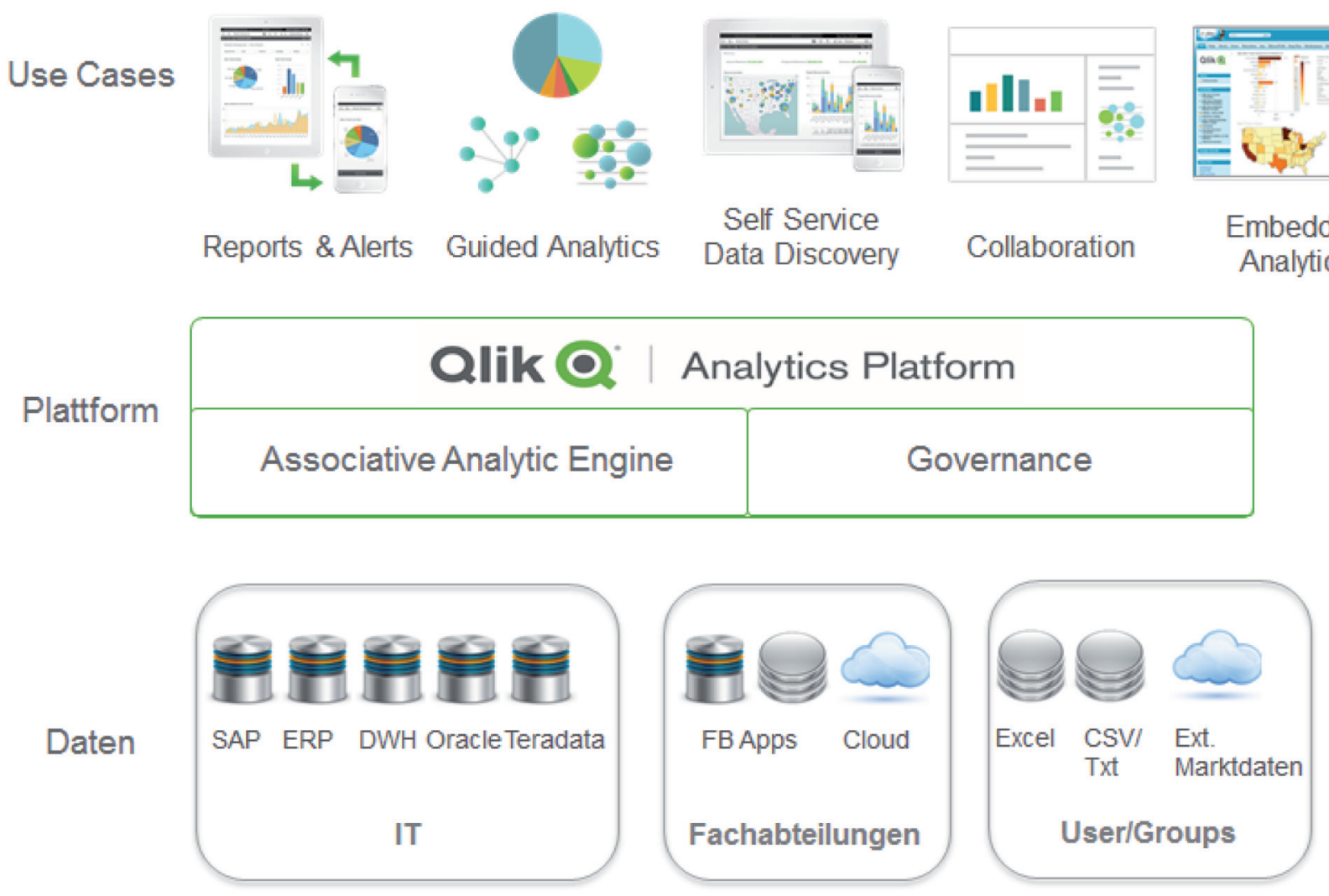

Abbildung 1: Visual Analytics Platform. Quelle: Qlik.

spektiven betrachtet werden können. Datenanalyse darf nicht aus vorgefertigten Bausteinen oder starren Reports bestehen, sondern muss dem Anwender die Möglichkeit bieten, neue Erkenntnisse zu gewinnen. Diese Eigenständigkeit darf jedoch nicht zu Lasten von Sicherheit und Datenschutz im Unternehmen gehen. Denn Agilität in der Auswertung darf nicht zur Anarchie bei den Zugriffsrechten führen: Es muss klar definiert sein, wer Zugriff hat, wie weit dieser Zugriff geht und in welcher Form mit den Daten gearbeitet werden darf. Richtlinien guter Unternehmensführung, die alle Daten und Nutzergruppen einschließen, gehören zu einer Visual Analytics-Plattform deshalb genauso dazu wie Kreativität und Freiheit. Bei diesem Sicherheitsmodell geht es nicht nur um die Frage, wer Zugriff auf Daten hat, sondern auf welche Daten genau und was sie damit machen: ein flexibles Policy-basiertes Framework, eine wichtige Basis für intuitive Self-Service-Analysen.

\section{Offenheit der Lösung}

Zeitgemäße Lösungen sind nicht auf einen Hersteller fokussiert. Das gilt sowohl für die Daten als auch für ihre Aufbereitung. Spezifische Daten, die unternehmensintern nicht vorhanden sind, aber einen wichtigen Mehrwert zur Entscheidungsfindung bieten, sollten problemlos aus öffentlichen Quellen ergänzt werden können. Offene Standard-APIs sorgen dafür, dass Datenanalysen mit neuesten Web-Technologien kombiniert werden können. Dazu gehören JavaScript, HTML5, CSS3 und Node. Entwickler können über Mashup- und Erweiterungs-APIs schnell und einfach komplexe Webanwendungen und benutzerdefinierte Visualisierungen erstellen.

\section{Die Entscheidungsmacht liegt beim Anwender}

Letztendlich darf nicht vergessen werden: Daten sind nichts anderes als eine Quelle für die Entscheidungsfin- 
dung. Visualisierungen unterstützen dabei, komplexe Sachverhalte schneller zu verstehen und leichter im Gedächtnis zu behalten. Es liegt an den Menschen im Unternehmen, Zusammenhänge zwischen den Daten zu verstehen und darauf basierend Entscheidungen zu treffen. Ziel einer Visual Analytics-Plattform ist es daher, alle Mitarbeiter eines Unternehmens durch Informationsbereitstellung zu ermächtigen, aus Daten neue Erkenntnisse und neues Wissen zu gewinnen und so Entscheidungen so schnell wie möglich treffen zu können.

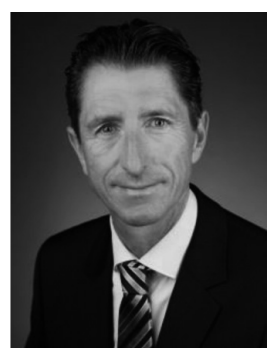

Alexander Klaus

QlikTech GmbH

Tersteegenstraße 25

40474 Düsseldorf

alexander.klaus@qlik.com

www.qlik.com/de

Deskriptoren: Datenanalyse, Maschinell, Visualisierung,

Systemumgebung, Benutzung

Alexander Klaus ist Marketingdirektor für die DACH-Region bei dem Visual-Analytics Anbieter Qlik. 